УДК 621.313 1:621.3.045.532

05.00.00 Технические науки

ИНФОРМАЦИОННЫЕ ПРИЗНАКИ
ПОВРЕЖДЕНИЯ ОБМОТКИ СТАТОРА ДЛЯ
ПОСТРОЕНИЯ РЕЛЕЙНОЙ ЗАЩИТЫ
АВТОНОМНОГО АСИНХРОННОГО
ГЕНЕРАТОРА

Богдан Александр Владимирович д.т.н., доцент, профессор кафедры SPIN-код6662-4171, asob2010@mail.ru

Соболь Александр Николаевич

к.т.н., доцент кафедры ФГБОУ ВПО Кубанский государственный аграрный университет, Краснодар, Россия

В большинстве случаев (85-95\%) отказы асинхронных машин происходят из-за повреждения статорной обмотки. При этом более $90 \%$ повреждений приходится на межвитковые замыкания. Замыкание небольшого количества витков статорной обмотки автономного асинхронного генератора не существенно изменяет основной магнитный поток машины, и, поэтому велика вероятность его длительной работы с таким видом повреждения. Скрытый отказ, существующий в виде виткового замыкания, значительно снижает надежность генератора, как источника резервного питания. На данный момент чувствительных защит асинхронных генераторов не существует, так как существует мнение, что в случае коротких замыканий (КЗ) генератор теряет возбуждение, и защита для него не требуется. Выявление информационных признаков витковых коротких замыканий в обмотке статора асинхронного генератора позволяет разработать релейную защиту. Основным принципом обнаружения витковых замыканий в обмотке статора асинхронного электродвигателя является измерение появляющейся несимметрии токов статора. Установлено, что в случае витковых КЗнесимметрия токов и напряжений не велика и сравнима с несимметрией в цепи нагрузки ААГ. Это не позволяет выполнить защиту, реагирующую на данную несимметрию. Тоже можно сказать и об изменении гармонического спектра токов и напряжений. Для построения защиты необходимо применять высокочувствительные схемы для обнаружения несимметрии трехфазной системы токов и напряжений, а также искажения формы токов

Ключевые слова: РЕЛЕЙНАЯ ЗАЩИТА, ВИТКОВЫЕ ЗАМЫКАНИЯ, ОБМОТКА СТАТОРА, АВТОНОМНЫЙ АСИНХРОННЫЙ
UDC 621.313 1:621.3.045.532

Technical sciences

\section{ESTIMATES OF THE ERROR OF \\ EXPERIMENTAL DATA AT STUDIES OF DENSITY AND THE SATURATED VAPOR PRESSURE (SVP) PETROLEUM PRODUCTS}

Bogdan Alexander Vladimirovich

Doctor of technical sciences, associate professor, professor of the chair, SPIN-code 6662-4171, asob2010@mail.ru

SobolAlexanderNikolaevich

Candidate of technical sciences, associate professor of the chair

Kuban State Agrarian University, Krasnodar, Russia

It is estimated that (85-95\%) of the electric motor failures occur due to damage to the stator winding. In this case, more than $90 \%$ of damage are because of interturn short circuits. Short circuits in stator windings of stand-alone asynchronous generator change the base magnetic flux incidentally, so the probability of its long work with such kind of damage is great. A latent failure, existing as a loop closure, significantly reduces the reliability of the generator as a backup power source. Now sensitive protections of asynchronous generators do not exist, since it is believed that in the case of short circuits (short circuit), the generator loses its excitation, and protection is not required for it. Identification of information signs of short-circuit currents in the stator winding of an asynchronous generator makes it possible to develop relay protection. The main principle of detection of stokes closures in the stator winding of an asynchronous electric motor is the measurement of the emerging asymmetry of the stator currents. It has been established that in the case of short-circuit faults, the asymmetry of the currents and voltages is not large and comparable to the asymmetry in the AAG load circuit. This does not allow performing a protection that reacts to this asymmetry. You can also say about changing the harmonic spectrum of currents and voltages. To build protection, it is necessary to use highly sensitive circuits for detecting the asymmetry of a three-phase system of currents and voltages, as well as distortion of the shape of currents and voltages, for example, by harmonic analysis. Do not exclude the possibility of using other protection devices, such as reacting the vibration of the case of an induction generator in the event of damage to the stator winding

Keywords: RELAY PROTECTION, SHORT CIRCUITS OF STATOR WINDINGS, STANDALONE ASYNCHRONOUS GENERATOR, 
ГЕНЕРАТОР, ГАРМОНИЧЕСКИЕ СОСТАВЛЯЮЩИЕ, ВИБРАЦИЯ

Doi: $10.21515 / 1990-4665-131-101$

\section{1. Введение}

В течение последних лет в нашей стране исследования и практика показали, что возможность применения автономных асинхронных генераторов (ААГ) в качестве альтернативных источников электроснабжения сельскохозяйственных предприятий, а также для личных нужд имеет достаточно большие перспективы [1]. Асинхронные генераторы различаются по способу возбуждения, характеру частоты (постоянная, изменяющаяся), способу стабилизации напряжения, конструктивному исполнению (с короткозамкнутым, фазным, полым ротором), числу фаз.ААГ с емкостным самовозбуждением имеет простую конструкцию, высокую надежность, относительно небольшую стоимость, является бесконтактной машиной.Автономный асинхронный генератор с емкостным самовозбуждением можно использовать для питания асинхронных электродвигателей, электробытовых приборов, для нужд освещения, обогрева и т. д. в районах, удаленных от линий электропередач и электростанций местного значения.

Значительный вклад в науку и практику их применения внесли такие ученые, как Н.И. Алиев, Н.Д. Торопцев, В.Я. Беспалов, М.Л. Костырев, А3. Р. Джендубаев и др. [7] Однако, при эксплуатации ААГ под воздействием различных факторов снижается электрическая прочность изоляции обмотки, что может привести к ее повреждению. Известно, что в большинстве случаев (85-95 \%) отказы асинхронных машин происходят из-за повреждения статорной обмотки. При этом более 90 \% повреждений приходится на межвитковые замыкания [2]. 
На данный момент чувствительных защит ААГ не существует, так как существует мнение, что в случае коротких замыканий (КЗ) ААГ теряет возбуждение, и защита для него не требуется [6].

Проверка этого положения, а также установление информационных признаков витковых коротких замыканий в обмотке статора ААГ для построения релейной защиты осуществлялась экспериментально.

\section{2. Постановка задачи}

Существует мнение, что АГ не требует защиты, так как при КЗ он теряет возбуждение. Проверка этого положения осуществлялась экспериментально.

Защиты электрических машин, в основном, строятся на изменениях: величин токов и их симметричных составляющих, величины и фазы тока (дифференциальные защиты), гармонических составляющих токов и магнитных потоков рассеяния, вибрации. Защиты генераторов применяются в основном для синхронных генераторов большой и средней мощности. Для построения собственной защиты ААГ необходимо знать специфику процессов в нем в случае витковых и межфазных КЗ в обмотке статора [3].

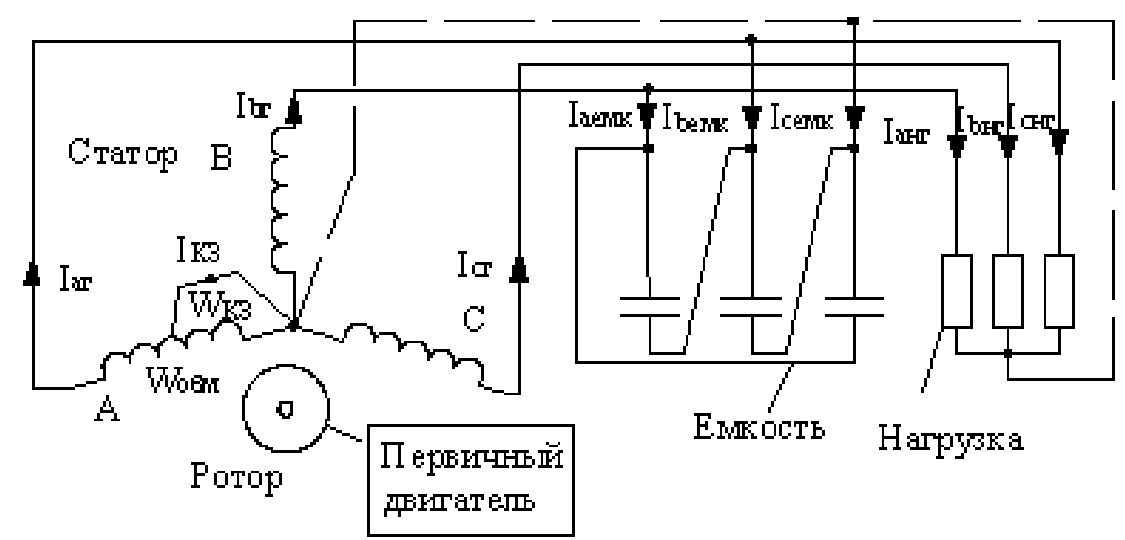

Рисунок 1 - Схема экспериментальной установки

Для эксперимента была подготовлена установка с АГ, выполненная на базе асинхронного электродвигателя с короткозамкнутым ротором типа 
4A100S4У3 (3 кВт, 1435 об/мин). [2] Схема установки изображена на рисунке 1. Емкость самовозбуждения равна 40 мкФ. Генератор приводился во вращение асинхронным двигателем. Искусственные замыкания между различными витками в фазных обмотках автономного асинхронного генератора создавались с помощью специальных, предварительно выведенных наружу из лобовой части обмотки статора, выводов.

\section{3. Результаты экспериментальных исследований}

Для анализа несимметрии токов и напряжений ААГ в случае витковых КЗ введем понятие коэффициента обратной последовательности токов $\kappa_{2}$ согласно [4]

$$
\mathrm{K}_{2}=\frac{\mathrm{I}_{2}}{\mathrm{I}_{1}},
$$

где $\mathrm{I}_{2}-$ ток обратной последовательности; $\mathrm{I}_{1}$ - ток прямой последовательности.

Также можно записать следующее выражение [4]

$$
\mathrm{K}_{2}=\frac{\sqrt{2} \sqrt{\left(\mathrm{I}_{\mathrm{A}}-\mathrm{I}_{\mathrm{C}}\right)^{2}+\left(\mathrm{I}_{\mathrm{C}}-\mathrm{I}_{\mathrm{B}}\right)^{2}+\left(\mathrm{I}_{\mathrm{B}}-\mathrm{I}_{\mathrm{A}}\right)^{2}}}{\mathrm{I}_{\mathrm{A}}+\mathrm{I}_{\mathrm{B}}+\mathrm{I}_{\mathrm{C}}},
$$

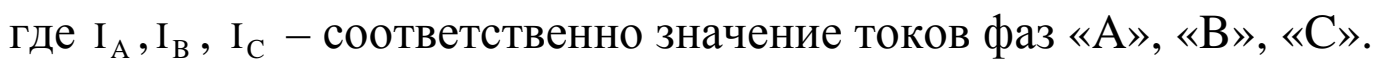

На рисунке 2 показаны кривые изменения токов КЗ генератора с приводом при $\mathrm{n}=$ const (Ікз1) и $\mathrm{n}=\mathrm{var}$ (Ікз2) от процентного соотношения числа замкнутых витков к числу витков в фазе ( w )

$$
\mathrm{W}=\frac{\mathrm{W}_{\mathrm{K} 3}}{\mathrm{~W}_{\text {обм }}},
$$

где $\mathrm{w}_{\text {кз }}$ - число замкнутых витков; $\mathrm{w}_{\text {обм }}$ - число витков в фазе. 


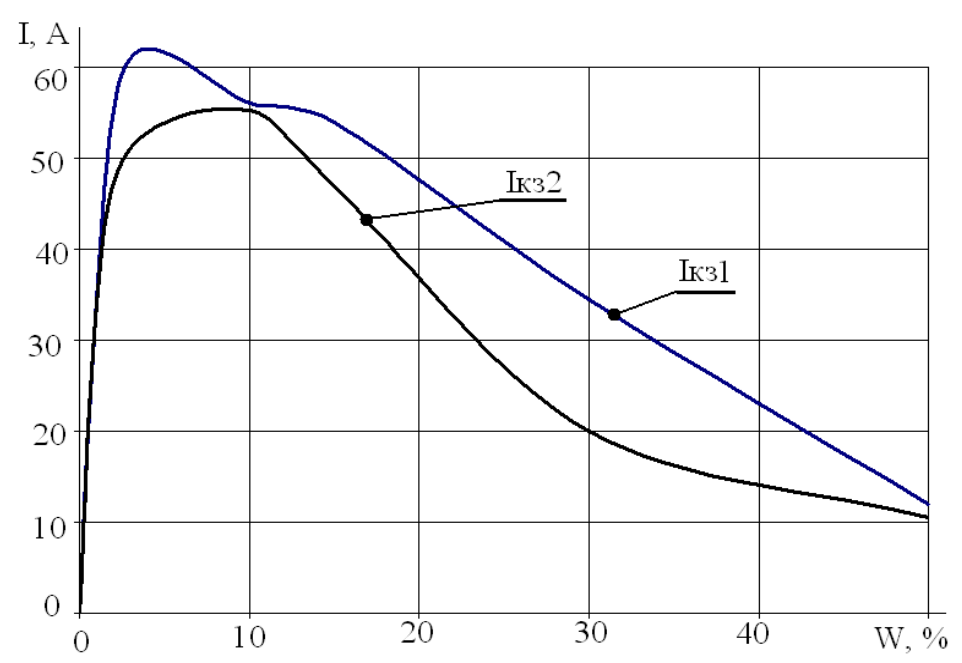

Рисунок 2 - Кривые изменения токов КЗ генератора

На рисунке 3 показаны кривые изменения токов генератора Iaг, Ibг, Ісг в амперах (A) для фаз “A”, “B”, “C” соответственно, а также ток короткого замыкания Ікз в фазе “А” при активной нагрузке генератора 600Вт в зависимости от числа замкнувшихся витков по отношению к числу витков в фазе

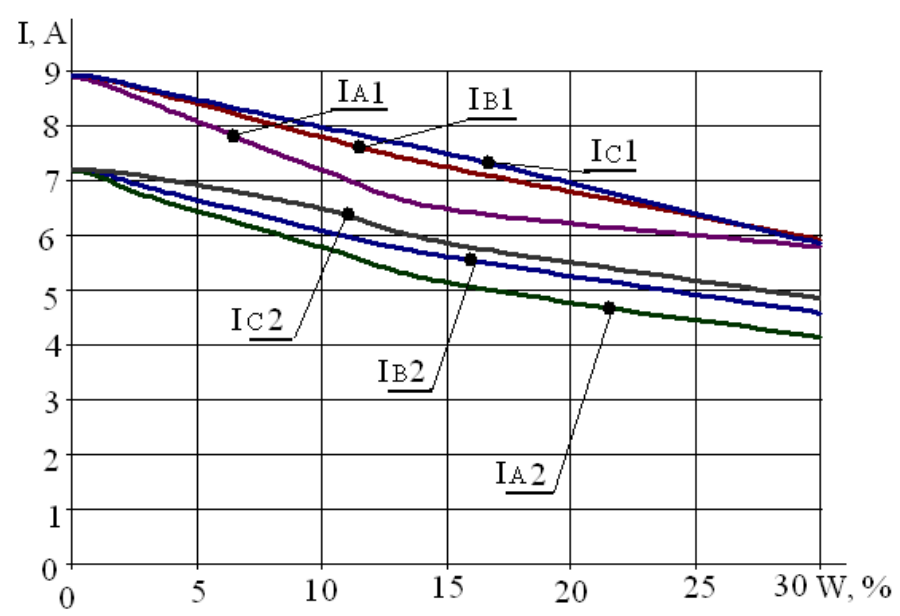

Рисунок 3 - Кривые изменения фазных токов генератора

Опыты показали, что при витковых и междуфазных КЗ в обмотке статора при малом числе замкнувшихся витков АГ не теряет возбуждения. Из полученных данных видно, что ток в короткозамкнутой части обмотки увеличивается в 5-10 раз по сравнению с номинальным. 
В случае 15 \% К3 витков фазные, емкостные токи, напряжения в поврежденной фазе уменьшаются в 1,3 раза (привод $\omega=$ const и чисто активная нагрузка). Коэффициент обратной последовательности для фазных, емкостных токов и фазных напряжений имеет величину менее 0,1 . Разница между средними значениями токов и напряжений фаз при $15 \%$ К3 витков, и токами и напряжениями в поврежденной фазе, по отношению к номинальным величинам, будет соответственно 0,04 $\mathrm{I}_{\text {ном }}$ и 0,07 $\mathrm{U}_{\text {ном }}$. Ток нагрузки в поврежденной фазе в этом случае уменьшается в 1,1 раза. Коэффициент обратной последовательности имеет величину менее 0,05.

Таким образом, большой несимметрии фазных токов нагрузки не наблюдается. Генератор теряет возбуждение только при 15-30 \% замкнутых витков.

Кривые изменения емкостных токов ААГ представлены на рисунке 4.

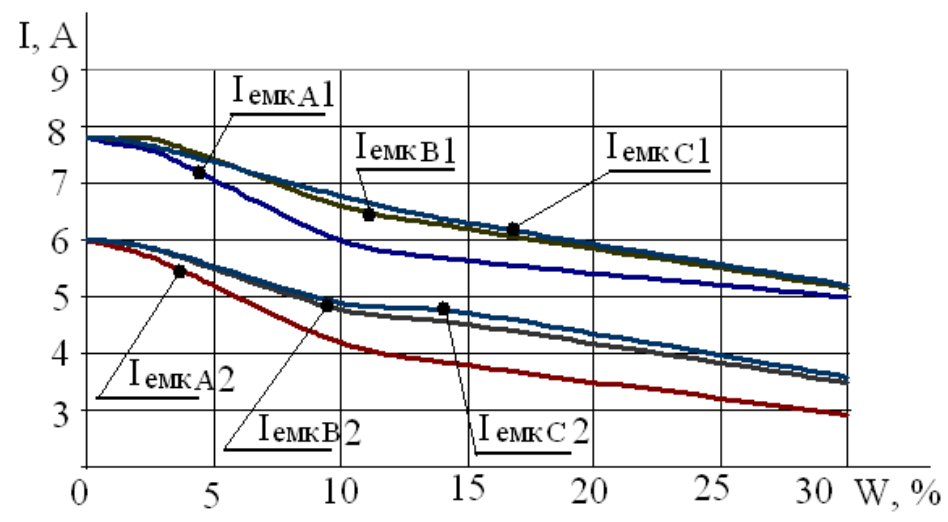

Рисунок 4 - Кривые изменения емкостных токов генератора

На рисунке 5 приведены осциллограммы тока и напряжения в поврежденной фазе А, соответственно емкостного тока, тока нагрузки, а также тока короткого замыкания при замыкании $15 \%$ витков обмотки статора и осциллограмма изменения сигнала с датчика вибрации, прикрепленного к ААГ. 
Из полученных осциллограмм видно, что при КЗ происходит уменьшение тока и напряжения в поврежденной фазе, также соответственно емкостного тока, тока нагрузки. При этом в момент замыкания наблюдается характерный всплеск фазного, емкостного токов, а также тока в короткозамкнутом контуре. Далее в течение небольшого промежутка времени токи принимают установившиеся значения. При уменьшении напряжения и тока нагрузки данного характерного всплеска не наблюдается. Изменение токов в неповрежденных фазах аналогичное.

В случае междуфазных (двухфазных и трехфазных) КЗ внутри обмотки статора также происходит уменьшение соответствующих величин и наблюдается характерный всплеск [2]. Однако, при симметричном замыкании пиковые значения токов при всплеске примерно одинаковые, а при несимметричном замыкании всплеск тока в фазе с большим числом замкнутых витков заметно больше, чем в остальных фазах. Ток КЗ в случае симметричного повреждения больше, чем при несимметричном повреждении.

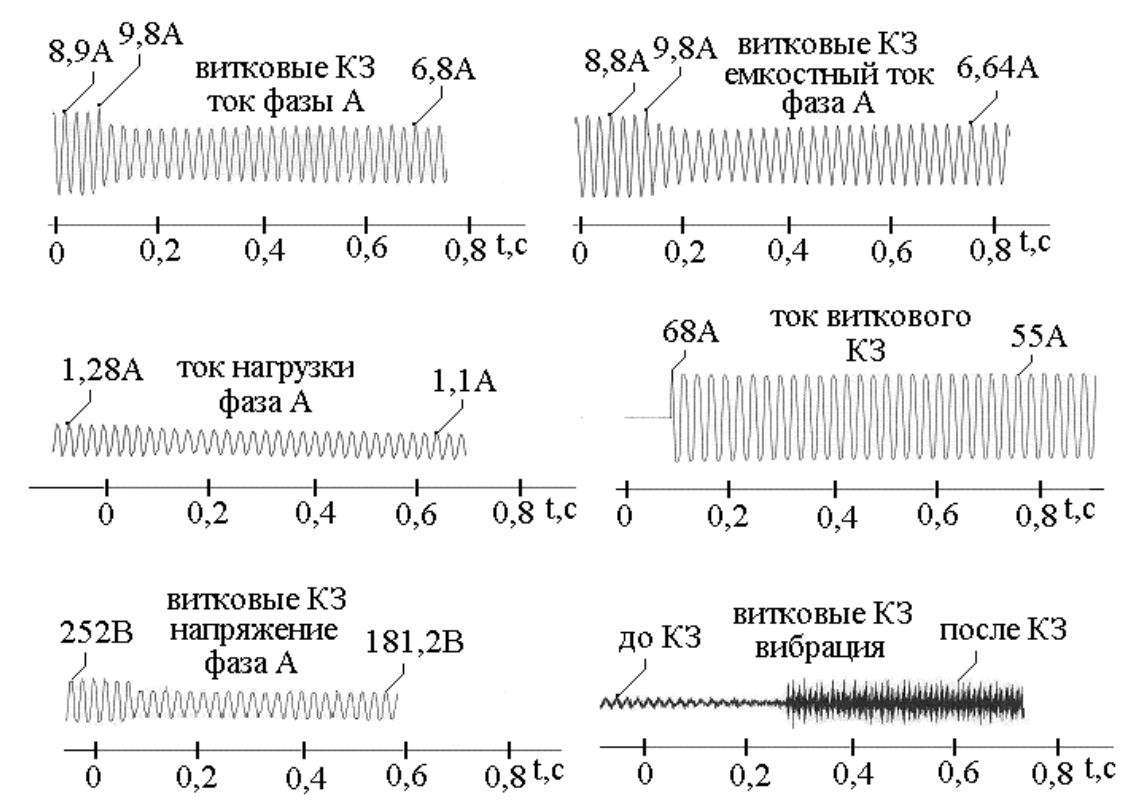

Рисунок 5 - Осциллограммы при витковом замыкании 
Также было установлено изменение гармонического состава соответствующих величин при витковом КЗ. На рисунке 6 показан спектр фазных токов ААГ при витковом замыкании $15 \%$ витков в фазе «А» и чисто активной нагрузке (для системы $\omega=$ const).

В ходе проведения экспериментальных исследований установлено, что при $15 \%$ КЗ витков наблюдается уменьшение первой, пятой и седьмой гармоник фазных и емкостных токов в поврежденной фазе соответственно в 1,15, 2,15 и 2,9 раз, а также рост третьей гармоники в 1,9 раз.

Для фазных напряжений происходит уменьшение первой, третьей, пятой и седьмой гармоник в поврежденной фазе соответственно в 1,53, $1,68,1,43$ и 1,1 раза.

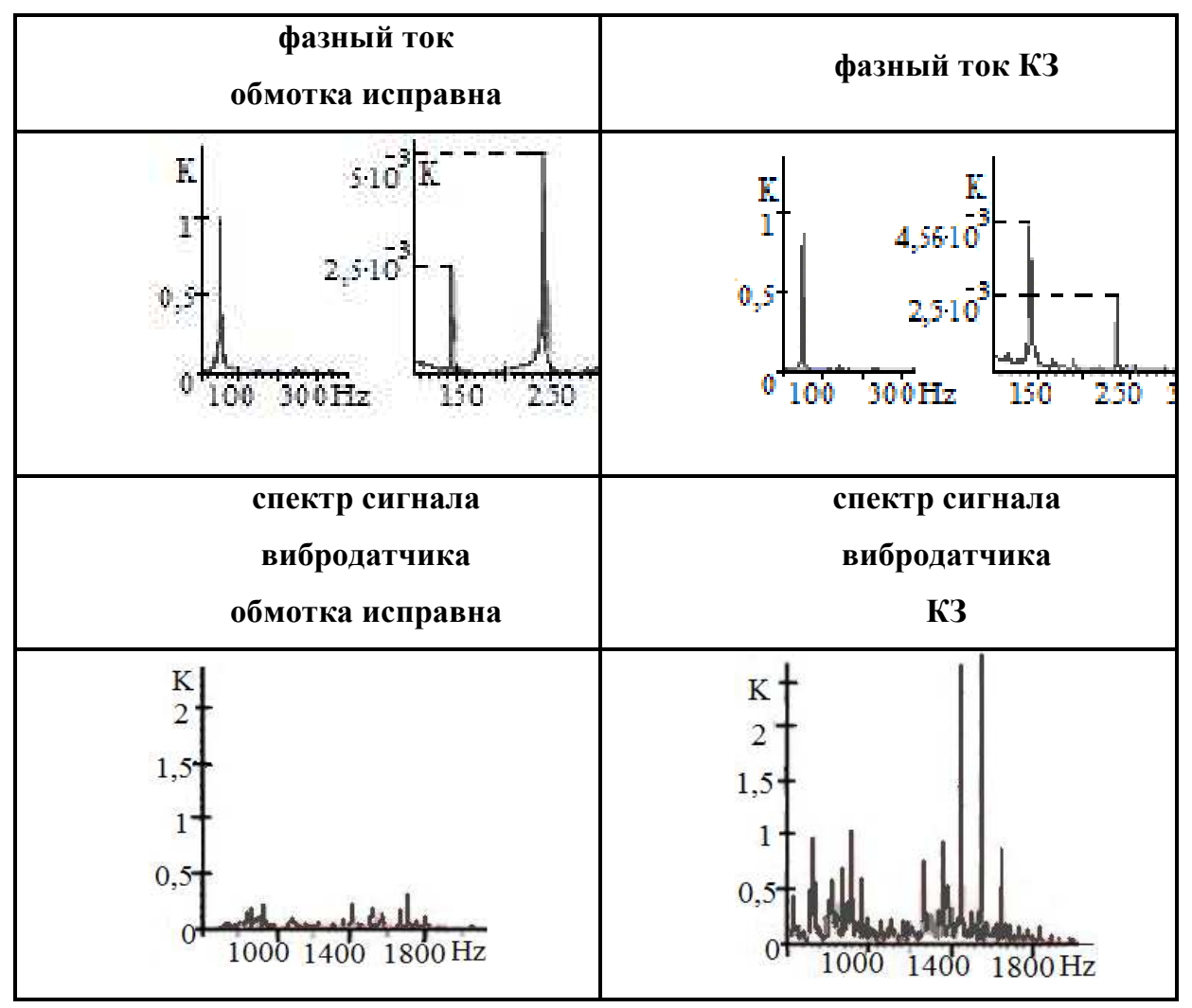

Рисунок 6 - Спектральный состав при 15 \% КЗ витков в фазе «А» 
Подобно напряжению происходит уменьшение данных гармоник тока нагрузки, но в 1,4 раз меньше.В целом же была установлена несимметрия гармонических составляющих, аналогичная несимметрии токов и напряжений в фазах.

Аналогичный характер имеет изменение гармонических составляющих в неповрежденных фазах. Изменение гармонических составляющих токов и напряжений в случае межфазных и трехфазных несимметричных КЗ аналогично изменению при витковых КЗ. При симметричном трехфазном КЗ наблюдается рост первой гармоники фазных и емкостных токов в 1,25 раз. Третья, пятая и седьмая гармоники уменьшаются в 2,8, 1,5 и 1,1 раз. Аналогичные соотношения получены для фазных напряжений, однако в данном случае первая гармоника уменьшается, а третья возрастает. Гармонические составляющие для токов нагрузки уменьшаются подобно напряжению, но в 1,4 раз меньше.

\section{4. Выводы и возможность использования экспериментальных данных для построения релейной защиты}

Таким образом, можно сделать вывод, что замыкание малого числа витков не ведет к развозбуждению генератора и может приводить к термическому повреждению обмотки статора, пожару и т.д.

Основным принципом обнаружения витковых замыканий в обмотке статора асинхронного электродвигателя является измерение появляющейся несимметрии токов статора. Установлено, что в случае витковых КЗнесимметрия токов и напряжений не велика и сравнима с несимметрией в цепи нагрузки ААГ. Это не позволяет выполнить защиту, реагирующую на данную несимметрию. Тоже можно сказать и об изменении гармонического спектра токов и напряжений.

Поэтому для построения защиты необходимо применять высокочувствительные схемы для обнаружения несимметрии трехфазной 
системы токов и напряжений, а также искажения формы токов и напряжений например, путем гармонического анализа.

Не исключается возможность использования других устройств защиты, например реагирующих на несимметрию магнитного поля внутри генератора в случае виткового КЗ в статорной обмотке (при размещении кольца, охваченного ферромагнитным сердечником, внутри электрической машины) [5].

Однако, размещение кольца внутри машины сопряжено с необходимостью разборки и сборки машины и представляет сложную технологическую операцию. Наводимое в кольце электромагнитное поле не дает точного представления о характере нарушения изоляции и возникновении короткого замыкания в обмотке, что приводит к необходимости усложнения схемы блока защиты. Также сложность блока защиты не позволяет обеспечить его высокую надежность. Тоже можно сказать и об устройствах тепловой защиты.

Для защиты ААГ от внутренних межфазных КЗ в обмотке статора, вблизи нулевых выводов можно использовать устройство дифференциально-фазной защиты, работа которого основана на измерении токов фаз в случае К3 [8]. Также можно использовать устройство дифференциальной защиты ААГ, работа которого основана на измерении токов в нулевых, фазных выводах и присоединении емкостей [10].

В качестве чувствительного устройства защиты, позволяющего определять и витковые замыкания обмоток статора ААГ, имеется возможность использовать устройство, основанное на использовании вибродатчика, который крепится на корпусе генератора [9].

На рисунке 7 показана функциональная схема управления и защиты $\mathrm{AA \Gamma}$ 


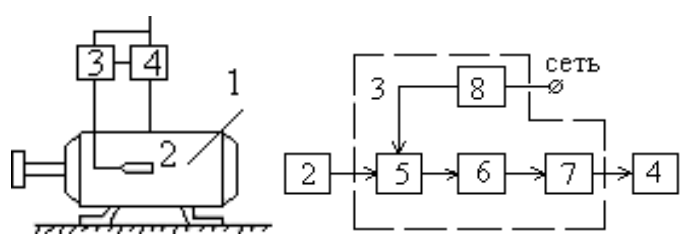

Рисунок 7 - Схема защиты ААГ на основе вибродатчика

При возникновении виткового КЗ в обмотке статора и при неисправности подшипников появляются дополнительные колебания корпуса машины 1, и сигнал вибродатчика 2 превышает пороговое значение. Сигнал на выходе блока сравнения 5 усиливается усилителем 6 и вызывает срабатывание исполнительного механизма 7 УЗ. Исполнительный механизм 7 приводит в действие привод аварийного отключения выключателя 4, который отключает ААГ от цепи емкостей самовозбуждения. Блок питания 8 подключен к блокам 5 и 6 и обеспечивает работу этих блоков. Блоки 5, 6, 7 и 8 в совокупности составляют УЗ 3.

\section{Список литературы}

1. Богдан А.В., Диагностика повреждений обмотки статора автономного асинхронного генератора / А.В. Богдан, А.Н. Соболь // Известия высших учебных заведений. Электромеханика, 2013. -№ 1.- С.70 - 71.

2. Богдан А. В. Признаки повреждения обмотки статора асинхронного генератора / А.В. Богдан, И.А. Потапенко, А.Н. Соболь // Механизация и электрификация сельского хозяйства, 2007.- № 8. -С.13 - 14 .

3. Гашимов М.А. Исследование в целях диагностики физических процессов функционирования электрических машин при неисправностях в обмотке статора и ротора / М.А. Железко, С.В. Абдулзаде // Электротехника, 2004.- № 2.- С. 20-27.

4. Железко Ю.С. Компенсация реактивной мощности и повышение качества электроэнергии / Ю.С. Железко. - М.: Энергоатомиздат, 1985.

5. Клецель М.А. Защита электродвигателей на катушках индуктивности от витковых замыканий / М.А. Клецель// Проекты и исследования, 1994.- № 3.- С. 17 - 20.

6. Соболь А.Н. Защита автономных асинхронных генераторов сельскохозяйственного назначения от витковых коротких замыканий: автореф. дис. кта техн. наук. - Краснодар: КубГАУ, 2010, 24 с.

7. Торопцев Н.Д. Области применения асинхронных генераторов / Н.Д. Железко // Энергетик, 2004. _№ 3.- С. 31 - 34.

8. Пат.2313890 РФ RU, МПК 51 H02M 7/08, Н02H 3/28. Устройство для дифференциально-фазной защиты.

9. Пат. № 2295815 РФ RU, МПК 51 H02H 7/08, G01M 15/00, H02K 15/00. Устройство защиты машин переменного тока. 
10. Пат. № 66127 РФ RU, МПК 51 H02K 11/00, Н02Н 7/08. Устройство для дифференциальной защиты асинхронного генератора.

\section{References}

1. Bogdan A.V., Diagnostika povrezhdenij obmotki statora avtonomnogo asinhronnogo generatora / A.V. Bogdan, A.N. Sobol' // Izvestija vysshih uchebnyh zavedenij. Jelektromehanika, 2013. № 1. S.70 - 71.

2. Bogdan A. V. Priznaki povrezhdenija obmotki statora asinhronnogo generatora / A.V. Bogdan, I.A. Potapenko, A.N. Sobol' // Mehanizacija i jelektrifikacija sel'skogo hozjajstva, 2007. № 8. S.13 - 14 .

3. Gashimov M.A. Issledovanie v celjah diagnostiki fizicheskih processov funkcionirovanija jelektricheskih mashin pri neispravnostjah v obmotke statora i rotora / M.A. Zhelezko, S.V. Abdulzade // Jelektrotehnika, 2004. № 2. S. 20-27.

4. Zhelezko Ju.S. Kompensacija reaktivnoj moshhnosti i povyshenie kachestva jelektrojenergii / Ju.S. Zhelezko. M.: Jenergoatomizdat, 1985.

5. Klecel' M.A. Zashhita jelektrodvigatelej na katushkah induktivnosti ot vitkovyh zamykanij / M.A. Klecel'// Proekty i issledovanija, 1994. № 3. S. 17- 20.

6. Sobol' A.N. Zashhita avtonomnyh asinhronnyh generatorov sel'skohozjajstvennogo naznachenija ot vitkovyh korotkih zamykanij: avtoref. dis. k-ta tehn. nauk. - Krasnodar: KubGAU, 2010, $24 \mathrm{~s}$.

7. Toropcev N.D. Oblasti primenenija asinhronnyh generatorov / N.D. Zhelezko // Jenergetik, 2004. _ № 3. S. $31-34$.

8. Pat.2313890 RF RU, MPK 51 H02M 7/08, H02H 3/28. Ustrojstvo dlja differencial'no-faznoj zashhity.

9. Pat. № 2295815 RF RU, MPK 51 H02H 7/08, G01M 15/00, H02K 15/00. Ustrojstvo zashhity mashin peremennogo toka.

10. Pat. № 66127 RF RU, MPK 51 H02K 11/00, H02H 7/08. Ustrojstvo dlja differencial'noj zashhity asinhronnogo generatora. 